\title{
A REVIEW OF RETINAL PROSTHESIS APPROACHES
}

\author{
TRAN TRUNG KIEN \\ School of Computer Science, The University of Nottingham Malaysia Campus, Jalan Broga, Semenyih, \\ Selangor 43500, Malaysia \\ khyx1ttk@nottingham.edu.my \\ TOMAS MAUL \\ School of Computer Science, The University of Nottingham Malaysia Campus, Jalan Broga, Semenyih, \\ Selangor 43500, Malaysia \\ Tomas.Maul@nottingham.edu.my \\ ANDRZEJ BARGIELA \\ School of Computer Science, The University of Nottingham, \\ Nottingham, $N G 81 B B$ \\ abb@cs.nott.ac.uk
}

\begin{abstract}
Age-related macular degeneration and retinitis pigmentosa are two of the most common diseases that cause degeneration in the outer retina, which can lead to several visual impairments up to blindness. Vision restoration is an important goal for which several different research approaches are currently being pursued. We are concerned with restoration via retinal prosthetic devices. Prostheses can be implemented intraocularly and extraocularly, which leads to different categories of devices. Cortical Prostheses and Optic Nerve Prostheses are examples of extraocular solutions while Epiretinal Prostheses and Subretinal Prostheses are examples of intraocular solutions. Some of the prostheses that are successfully implanted and tested in animals as well as humans can restore basic visual functions but still have limitations. This paper will give an overview of the current state of art of Retinal Prostheses and compare the advantages and limitations of each type. The purpose of this review is thus to summarize the current technologies and approaches used in developing Retinal Prostheses and therefore to lay a foundation for future designs and research directions.
\end{abstract}

Keywords: Retina; retinal prosthesis; vision; image processing; neural computation. 


\section{Retina Review}

People see the world based on the light that is reflected off objects. Light stimuli enter the eyes and are then converted into electrical stimuli by the retina. The electrical patterns generated by the retina represent an early and yet complex level of information processing. This low-level information is passed to higher brain regions, eventually leading up to a visual understanding of the environment. Based on the anatomical depiction in figure one, the retina consists of several layers, each one with its own name and functionality.

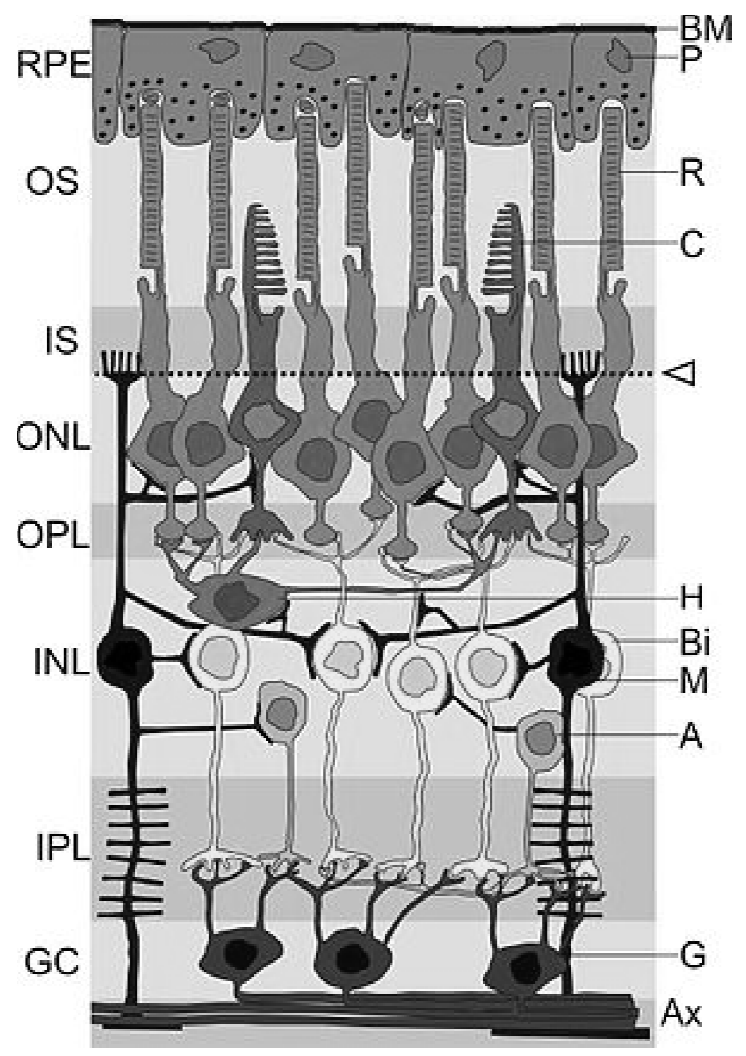

Fig. 1. The structure of the retina ${ }^{60}$

There are six main layers in the retina: Photoreceptor layer, outer nuclear layer, outer plexiform layer, inner nuclear layer, inner plexiform layer and ganglion cell layer. The layers are differentiated primarily based on their constituent cells and connections. In term of cell type, there are five major cells in the retina, which are: 
- Rods and cones,

These cells both sit within the photoreceptor layer and serve the same function of sensing light and converting it into electrical signals. These cell types also have morphological and functional differences. Rods are designed primarily for night (scotopic) vision and have only one type of cell in terms of wavelength sensitivity. On the other hand, there are three types of cones (red, green and blue) each one tuned to a different wavelength (i.e. L, M, S or long, medium and short wavelength) and designed primarily for day (photopic) vision.

- Horizontal cells,

This type of retinal cell lies in between the inner nuclear layer and the outer plexiform layer and connects to rods, cones and bipolar cells. Horizontal cells receive input primarily from photoreceptors and provide lateral inhibition to both bipolar cells and photoreceptors.

- Bipolar cells,

These cells lie inside the inner nuclear layer and transfer signals from rods, cones and horizontal cells to amacrine and ganglion cells.

- Amacrine cells,

In between the bipolar cells and the ganglion cells lie the amacrine cells of which there are many sub-types. This varied group of cells embodies a vast network of lateral connections, which subserve multiple signal shaping functions. These lateral connections involve primarily bipolar and ganglion cells.

- Ganglion cells.

These are the output cells of the retina. Ganglion cells receive analogue input (i.e. graded potentials) from bipolar and amacrine cells and convert them into digital signals (i.e. action potentials or spikes) which are sent to higher brain regions via the optic nerve.

In summary, the retina is a multilayer neural tissue that converts light signals into electrical signals that can be decoded by the brain. There are six layers in the retina and each layer consists of specific types of cells and/or connections. Retinal cells are connected to each other in complex ways, forming circuits that implement rich information processing channels. Because the retina is a complex information processing system, different types of damage to its circuits lead to different types of vision problems, some of which are discussed in the next section.

\section{Eye Diseases}

Every year millions of people become blind through eye diseases such as Age-Related Macular Degeneration (AMD) or Retinitis Pigmentosa (RP). There are nearly 700,000 new AMD cases every year in the US and about $10 \%$ of them are defined as legally blind ${ }^{1}$.

The origin of AMD is the abnormal aging of the retinal pigment epithelium (RPE). The formation of yellow drusen on the RPE and the proliferation of leaky vessels in the subretinal space are the major symptoms of AMD. In terms of vision, early AMD symptoms consist of a distorted field of view in the center, eventually leading to an 
almost complete loss of vision in the central 30 degrees. There are a number of treatments for AMD patients which concentrate on slowing down the AMD process but still no complete cure exists for this disease ${ }^{2}$.

An early Retinitis Pigmentosa symptom consists of poor night vision due to damaged $\operatorname{rods}^{3,4}$. Following this, cones are gradually damaged leading to loss of vision in the midperiphery. Eventually complete peripheral vision is lost. In some exceptions RP patients still have major vision in the foveal or parafoveal regions, while vision in the remaining parts of the retina is completely lost. There are over 100 defects that affect photoreceptors and there are still no treatments for $\mathrm{RP}^{5}$.

Although retinal cells are destroyed due to AMD or RP, some parts of the retina are still intact. For instance, in RP although only $4 \%$ of photoreceptors remain, about $30 \%$ of ganglion cells and $80 \%$ of the inner retina are still intact ${ }^{63}$. In AMD, the percentage is higher with $90 \%$ of the inner retina still intact ${ }^{1}$. Hence, AMD and RP do not completely degenerate the retina and the hope is that we can restore vision by using the remaining cells.

\section{Retina Prosthesis Review}

Retinal prostheses are devices that can help the blind partially restore those visual functions that were lost due to RP or AMD. This paper will classify the prostheses by two criteria: hardware and software. On the hardware side, the design, implementation and current results will be discussed. On the software side, we will discuss implementation issues and the importance of software in order to restore vision to the blind.

\subsection{Hardware aspect}

Prostheses are categorized into two major types: Extraocular, which means the device is placed outside the eye and intraocular, which is placed inside the eye.

\subsubsection{Extracortical}

The visual cortex of the brain is responsible for visual information processing and is located primarily at the back of the brain in the occipital lobe ${ }^{6}$. By using an electrode array that is implanted in the skull and that stimulates the visual cortex, one can hope to create visual perception or phosphenes allowing the blind to navigate in the world. Phosphenes in this context are the simple spots or flashes of light that are artificially created by electrode stimulation of visual neurons. Brindley and Dobelle implanted 50 electrodes over the occipital lobe in a volunteer to restore visual perception via the visual $\operatorname{cortex}^{8-13}$. The volunteer initially was unable to recognize any letter or number but after a short time of practice, it is reported that the patient could count fingers and recognize six inch square characters at five feet ${ }^{8}$. The volunteer was also able to use this system when travelling alone in the city metro. Moreover, six other volunteers had no epileptic 
symptoms or systemic problems after the implant but there is still a risk of infection which requires removal of the implant ${ }^{11-12}$.

On the downside, it is difficult for patients to recognize large characters because of the "bottleneck" caused by the information exceeding the limit of the visual tunnel. Other issues include: "halos" surrounding phosphenes, the inability to appreciate distinct phosphenes as well as the inability to control the number of phosphenes. These difficulties in part motivate research into intracortical stimulation ${ }^{9-12}$.

\subsubsection{Intracortical}

The Illinois Intracortical Visual Prostheses project and the Utah Electrode Array are two lines of research that are expected to overcome the limitations of surface cortical stimulation. By using smaller electrodes that can get closer to the target neurons, this type of prosthesis is expected to use lower current. The Illinois prosthesis uses 152 intracortical microelectrodes while the Utah Electrode Array contains 100 electrodes ${ }^{14,15,16}$. In animal tests and short-term human implants, the intracortical prosthesis demonstrated the ability to produce phosphenes with color properties. This type of prosthesis avoids all damaged parts of the early visual system (e.g. retina) ${ }^{7}$.

\subsubsection{Optic nerve prostheses}

One disadvantage of cortical prostheses is that visual maps are spread across large cortical areas, making it difficult for electrode arrays to completely cover them. The optic nerve has an advantage with regards to this point because the entire visual field is placed in one small area. One proposed approach for an optic nerve prosthesis uses spiral cuff electrodes to stimulate the optic nerve and create visual sensations ${ }^{7}$. As can be seen in figure two, the cuff wraps around the optic nerve (A) and stimulates it based on the signal received by a camera (B). Veraart et al. performed this type of prosthesis on a blind volunteer and obtained encouraging results. By using this cuff, the prosthesis can produce multiple phosphenes by customizing the stimulation parameters and provides the ability to interface with the optic nerve safely. The above mentioned blind volunteer was able to interact with the environment by demonstrating basic pattern recognition skills such as recognition of different shapes, line orientations and even letters in some cases ${ }^{64,17-19}$. 


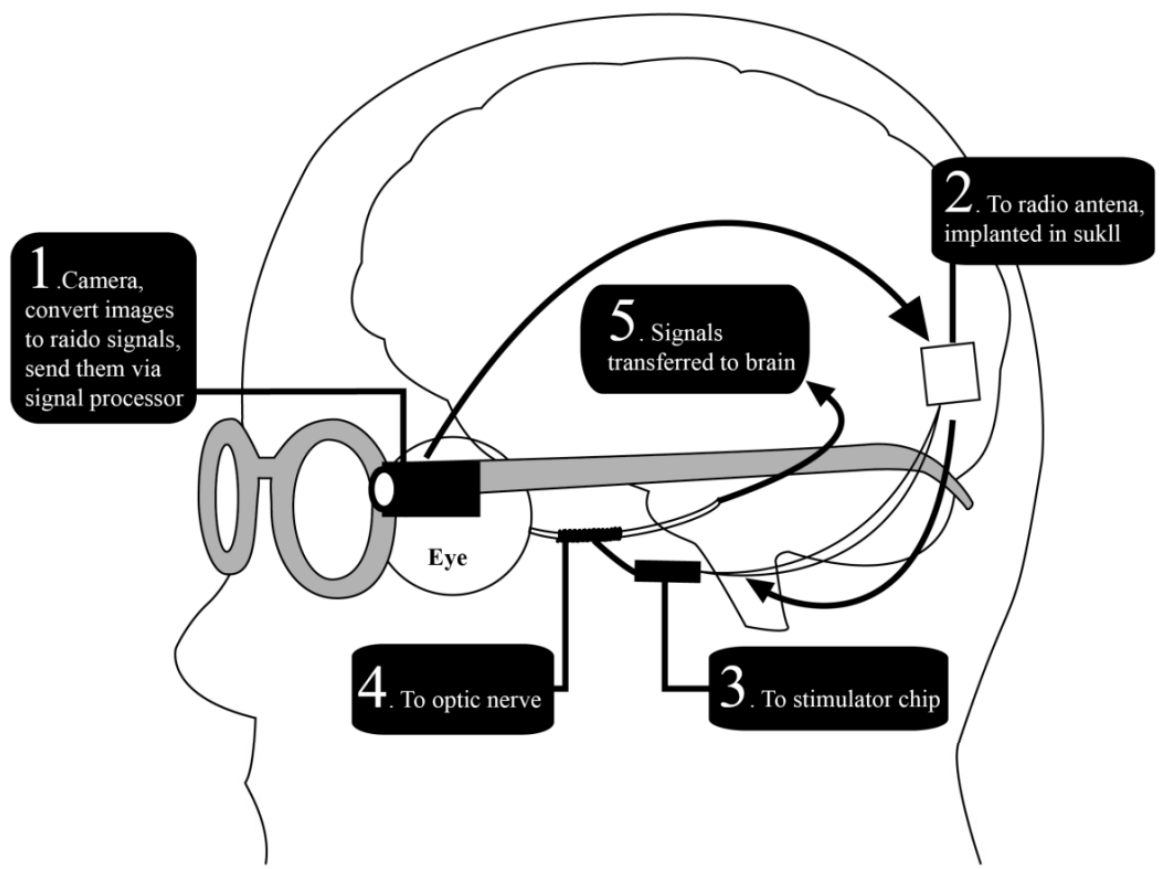

(A)

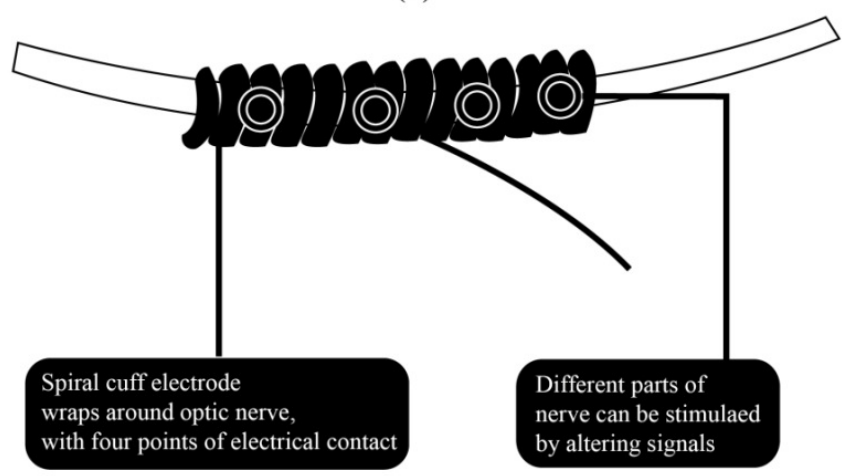

(B)

Fig. 2. The optic nerve prosthesis ${ }^{61}$

Although optic nerve prostheses provide some encouraging advantages, one significant problem related to cuff electrodes and its interface to the optic nerve ${ }^{7}$ consists of the fact that it requires a large number of contacts which can increase the risk of damage to the optic nerve ${ }^{7}$. In specific, the optic nerve implant requires the cut out of the dura mater which may infect the central nervous system or harm the blood flow inside the optic nerve ${ }^{13}$. Moreover, because of the density of the axons, precision in the specified stimulation is also reduced ${ }^{13}$. 


\subsubsection{Epiretinal prostheses}

Unlike extraocular devices, intraocular prostheses are devices that are implanted inside the eye thanks to several technologies such as: microelectronics, microelectronics packing, power and data telemetry as well as image processing. In general, intraocular prostheses belong to two major categories: epiretinal and subretinal. Each type has its own advantages and disadvantages. The structure and implementation of each category will be discussed in this section.

First category is epiretinal prosthesis, this device is implanted on the inner part of the retina, sitting closely on top of ganglion cells ${ }^{20}$. With the help of an external capture device such as a digital camera, the scene view is captured and transformed by the processing unit placed inside the eye and used to stimulate the remaining retinal neurons. Because of this process, this type of prosthesis requires power and data telemetry. There are many groups in the world that have tried to design as well as implement epiretinal prostheses in terms of external devices and internal processors to achieve the same goal of vision restoration. Based on the same requirements of maximizing the functionality of the remaining retinal tissue, minimizing power consumption and minimizing the number and size of devices implanted in the eye, various designs of epiretinal prostheses have been proposed and tested in animals as well as humans. Several typical examples of epiretinal prostheses will be discussed below.

The most mentioned epiretinal prosthesis is the Intraocular Retinal Prosthesis (IRP) by Mark Humayun, from the University of Southern California and private company Second Sight Medical products, Inc $^{7}$. 


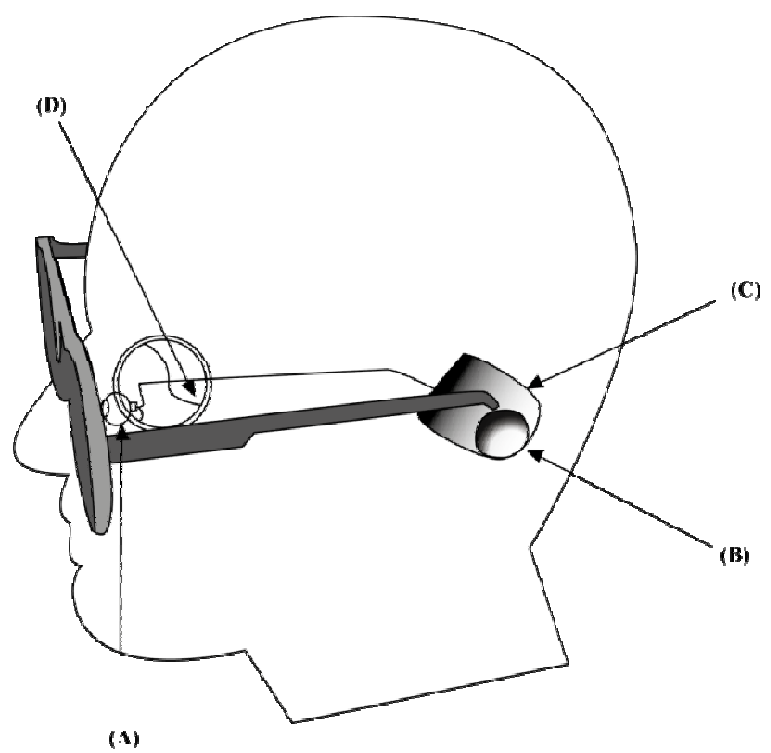

Fig. 3. The schematic of the prosthesis ${ }^{21}$

(A) Camera; (B) Wireless Transmitter; (C) Wireless Receiver; (D) Intraocular Implant

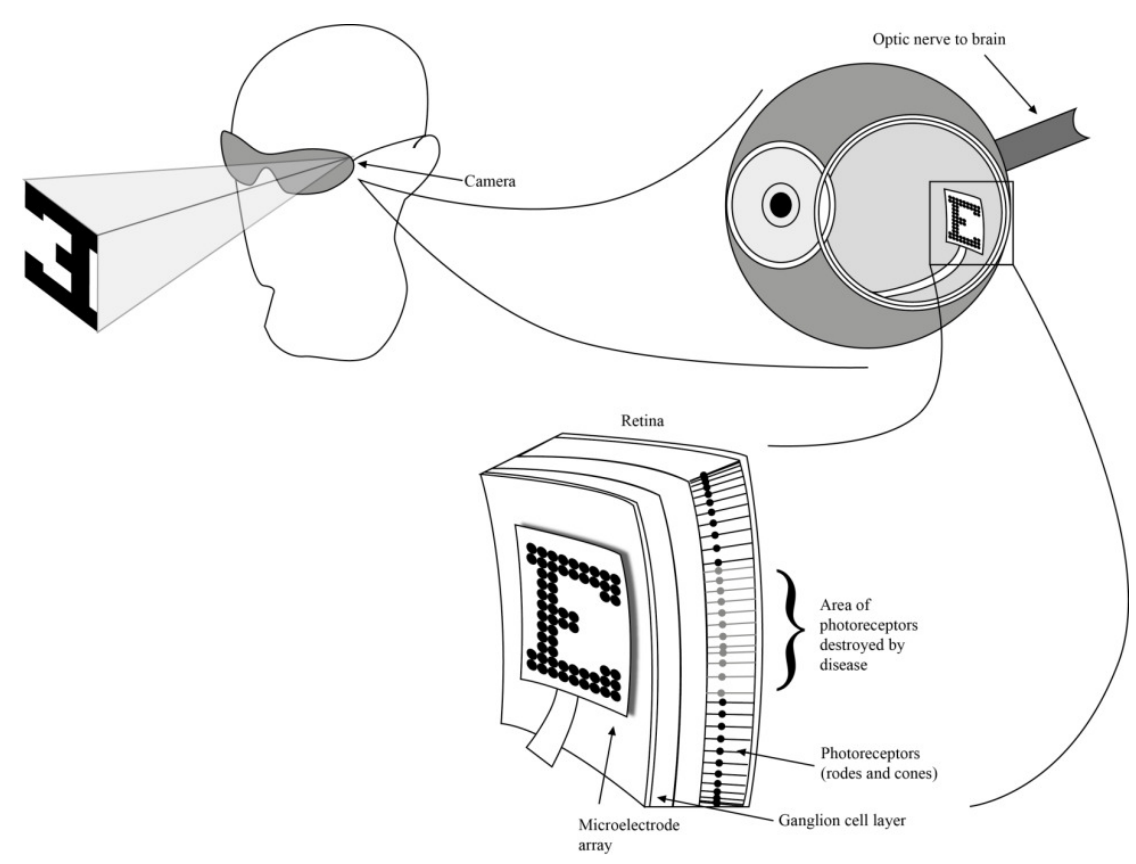

Fig. 4. The detail of the intraocular implant ${ }^{62}$ 
As can be viewed in figure three, two major parts of the prosthesis are an external camera mounted on a pair of glasses and an intraocular unit. The image is first captured by the camera, then analyzed and transformed by an image processing unit. The processed data is then transmitted to the prosthesis, which consists of 16 platinum microelectrodes which then stimulate the inner retinal neurons ${ }^{21,22}$. The illustration of the implant can be seen in figure four above.

After animal testing, the first version (Argus I) of the device was tested on humans and some encouraging results were obtained. All 16 electrodes were tested in terms of the threshold currents required for eliciting visual responses. Some of these thresholds were observed to change with time. In particular, in ten out of 16 electrodes the current thresholds remained constant, in three electrodes the threshold increased and in the remaining three the thresholds decreased. In addition, it was also observed that the size of the phosphenes increased when the stimulation current was higher ${ }^{24}$. Stable current helps the prosthesis save energy and increases its durability. The patients reported the experience of phosphenes and demonstrated the ability to discriminate between percepts created by different patterns of electrode stimulation. With the use of the camera, the patients were able to interact with the surrounding world and the more they practiced using the camera, the more they could tackle tasks of increasing complexity. Besides that, patients also reported experiencing four different colors as well as the "halo" effect. Moreover, after practicing for about two and a half months, patients were capable of differentiating ten levels of brightness as well as distinguishing the motion direction of objects $^{23,24}$.

The second version of the device (Argus II) ${ }^{25}$ incorporated some significant changes in hardware. The list of improvements can be seen below:

- 60 electrodes instead of 16 ,

- Wireless data transfer,

- MEMS components for better fixing of the device in the eye ball.

Argus II results from human trials are positive with patients capable of differentiating basic forms of motion, perceiving light and dark and even shooting baskets ${ }^{26}$. The third version of the prosthesis, Argus III, is currently under development. One of the main improvements here pertains to increased electrode resolution which promises to restore vision even further than previous versions ${ }^{62}$.

The second major effort in epiretinal prosthesis R\&D comes from Joseph Rizzo and John Wyatt at the Harvard Medical School ${ }^{7}$. The design of this device is the same as the IRP described above in terms of distinct intraocular and extraocular parts. The extraocular part consists of a CCD camera mounted on the pair of glasses and a battery. The intraocular component contains a photodiode panel and a stimulator chip which are placed on a modified lens. This prosthesis uses a laser pulse to transfer data from the camera to a stimulation chip instead of a cable as what is used in the first intraocular prosthesis (e.g. Argus I) ${ }^{27}$.

Experiments were conducted on three types of electrode arrays ${ }^{28}$ : 
- $10 x 10$ array of 50- $\mu \mathrm{m}$ diameter electrodes, with an inter-electrode spacing of $220 \mu \mathrm{m}$

- $8 \times 10$ array of 50- $\mu \mathrm{m}$ diameter electrodes, with an inter-electrode spacing of $220 \mu \mathrm{m}$

- $4 \times 5$ array of 100- $\mu \mathrm{m}$ diameter electrodes, with an inter-electrode spacing of $620 \mu \mathrm{m}$

The results of these experiments on five patients share similarities with those from Humayun et al. There was no damage after implantation and phosphenes were perceived in three patients when electrodes were stimulated with currents above threshold. Higher stimulation thresholds are suggested for patients with worse vision in order to stimulate the intact retinal cells ${ }^{28}$. Rizzo et al. tested eight hypotheses in blind patients ${ }^{28}$. For example, hypothesis two was verified with 48 trials and involved testing the correspondence between subject perception and different patterns of multi-electrode stimulation $^{27,28}$. Rizzo et al. noted that holding the electrodes above the retina surface makes the phosphenes elicited more consistent because areas with lower stimulation thresholds can be located more easily ${ }^{7}$. Due to significant disparities between the authors' hypotheses and their observations, Rizzo et al. have abandoned the epiretinal approach and are instead focusing on subretinal prostheses ${ }^{7}$.

The third effort in epiretinal prosthesis R\&D consists of The Learning Retina Implant, which was developed by 14 expert groups in Germany under the direction of Rolf Eckmiller since $1995^{7}$. Like the two former prostheses, this prosthesis contains an extraocular component or a retinal encoder (RE) that is placed on the pair of glasses or a contact lens. The retinal stimulator (RS) is the intraocular component which is placed on the epiretinal surface whereby contact is established with ganglion cells. The retinal encoder also has the responsibility of providing an integrative perception-based dialogue between the RE and human subject besides stimulating the complicated mapping operation of parts of the neural retina. The dialogue, which involves patients fine-tuning parameters of the encoding process, helps to provide ganglion cell with interpretable codes for epiretinal stimulation ${ }^{29}$.

Although testing the prosthesis on animals such as rabbits and primates has shown encouraging results ${ }^{30,31}$, some caution is being exercised in terms of human trials. One major issue being addressed in this work consists of how to develop an effective manmachine interface for the blind patient ${ }^{32,33}$. To tackle this issue, Eckmiller and his team chose to concentrate on the information processing challenge (brain, retina and retinal prosthesis) by adopting a dialogue-based RE tuning approach. Proper stimulation to elicit neural signals for the ganglion cells must be determined to optimize the dialog between the retinal encoder and the central visual system. Moreover, the desired percepts are generated by the central visual system only when the neural signals generated from the $\mathrm{RE}$ and $\mathrm{RS}$ are clear and unambiguous ${ }^{34,35}$. 


\subsubsection{Advantages and disadvantages of epiretinal prostheses}

- Advantages

The first advantage of the epiretinal approach is that the electrical device is mostly kept outside the retinal surface, keeping the heat at the retinal surface at low levels and hence making the prosthesis virtually harmless to the eye $\mathrm{e}^{36}$.

The second advantage is that the surgery process is easier. The surgical procedures involving the vitreous cavity are generally straightforward. Moreover, the device can be placed on the entire vitreous cavity in order to minimize disruption to the retina ${ }^{37}$. In general, epiretinal prostheses can be larger than subretinal prostheses.

The last advantage is that the approach does not require the remaining retinal cells (e.g. bipolar and amacrine cells) for information processing.

\section{- Disadvantages}

The first disadvantage of the epiretinal prosthesis is that the remaining retinal neural cells are not used to process the information ${ }^{38}$. This generally means that an external camera is required in order to allow for some degree of pre-processing in substitution of what would have been done automatically if the information had been passed through intact neurons such as bipolar and amacrine cells.

Secondly, in order to increase comfort and reduce retinal damage the shape and material of the electrode array substrate poses several challenges. The electrode array must be carefully fixed to the curved retinal surface in order for the stimulation to be consistent and to avoid retinal tearing ${ }^{37}$.

Finally, the image processing performed by the retina must be replicated in order for ganglion cells to be stimulated with meaningful signals ${ }^{37}$.

\subsubsection{Subretinal prostheses}

Subretinal prostheses are placed between bipolar cells and photoreceptor cells. Chow et al. developed a subretinal prosthesis consisting of thin silicon plates with two to three $\mathrm{mm}$ in diameter and 50-100 $\mu \mathrm{m}$ thickness as well as comprising several thousand lightsensitive micro-photodiodes. The device, named The Artificial Silicon Retina (ASR) ${ }^{38}$, is assembled by 5000 subunits, each one with dimensions $20 \times 20 \mu \mathrm{m}$. Each subunit is a combination of a micro-photodiode and a stimulating electrode and the density of the subunit on the plate is 1100 subunits per $\mu \mathrm{m}^{2}$. The function of each subunit is to convert light originating from the outside scene into electrical signals that can stimulate the remaining functional retinal cells. The solar cells are used to absorb and convert the light into electrical signals for stimulating and play role as photoreceptors. Besides that, the solar cells also provide the energy for the subunits by converting the light to electrical current. $^{39-42}$ 


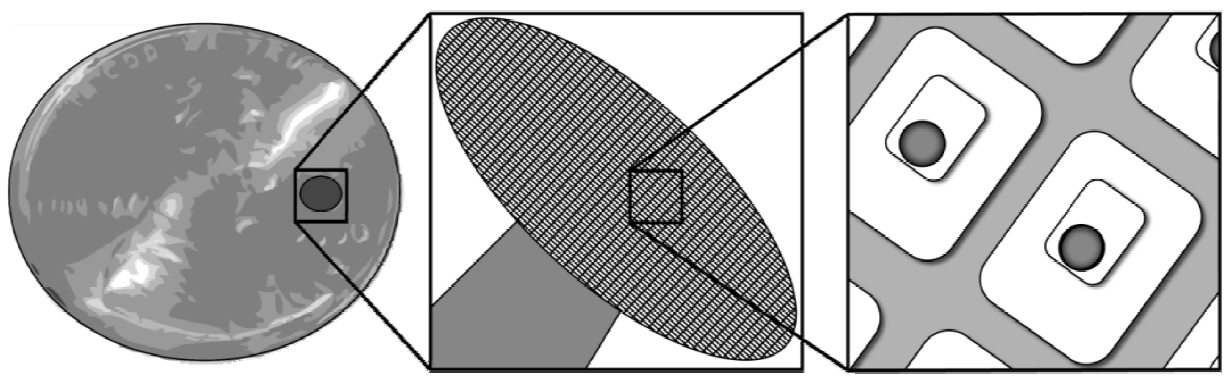

(A)

(B)

(C)

Fig. 5. The Artificial Silicon Retina (ASR) ${ }^{38}$

(A) ASR compared to a penny; (B) The ASR Microchip in closer view; (C) An ASR electrode.

The subretinal prostheses were implanted in six patients and the results were reported as follows ${ }^{38}$ :

Table 1. Result from testing the Artificial Silicon Retina ${ }^{38}$

\begin{tabular}{|c|c|c|}
\hline Patient & Eye Ability & Results \\
\hline Patient one & $\begin{array}{l}\text { Light Perception in both } \\
\text { eyes }\end{array}$ & $\begin{array}{l}\text { Capable of perceiving light without } \\
\text { heading to the light source }\end{array}$ \\
\hline Patient two & $\begin{array}{l}\text { Light Perception in left eye } \\
\text { only }\end{array}$ & $\begin{array}{l}\text { Capable of seeing shadows of people } \\
\text { with the right eye }\end{array}$ \\
\hline Patient three & $\begin{array}{l}\text { Had visual acuity of Hand } \\
\text { Moving }\end{array}$ & $\begin{array}{l}\text { Capable of using night light for } \\
\text { navigating and seeing movement on } \\
\text { television }\end{array}$ \\
\hline Patient four & $\begin{array}{l}\text { Had visual acuity of hand } \\
\text { Moving in both eyes }\end{array}$ & $\begin{array}{l}\text { Capable of navigating the yard and } \\
\text { locating light sources }\end{array}$ \\
\hline Patient five & $\begin{array}{l}\text { Had visual acuity of } \\
\text { counting fingers at } 1 \text { or } 2 \mathrm{ft} \\
\text { in both eyes }\end{array}$ & $\begin{array}{l}\text { Capable of differentiating paper } \\
\text { money, using utensils for eating and } \\
\text { recognizing faces }\end{array}$ \\
\hline Patient six & $\begin{array}{l}\text { Had a preoperative visual } \\
\text { acuity of Hand Moving in } \\
\text { both eyes and equal visual } \\
\text { function in both eyes }\end{array}$ & $\begin{array}{l}\text { Sometimes capable of recognizing } \\
\text { denominations of paper money } \\
\text { distinguishing traffic lights, locating } \\
\text { cars as well as finding a coffee cup a } \\
\text { meals. }\end{array}$ \\
\hline
\end{tabular}

Although the results of Chow et al.'s experiments are encouraging for blind patients, some weaknesses are also noted ${ }^{42,43}$. Specifically, the current generated by a microphotodiode is not sufficient to stimulate neurons due to its limited light-sensitivity. To overcome this issue, an external energy source is needed and only with the aid of this 
external power the light from the outside is sufficient to generate the stimulating current at each electrode. Moreover, Michael Javaheri et al. also reported that Chow et al. have abandoned the notion that the ASR Microchip is a prosthetic device and listed this prosthesis as a therapeutic device, not a truly retinal prosthesis ${ }^{7}$. This is mainly due to the power issues mentioned earlier. However, Chow et al.'s concept of a subretinal prosthesis, which is noted as the first of its kind, has inspired other researchers to develop better versions of subretinal prostheses.

Another subretinal prosthesis design was developed by Zrenner et al. ${ }^{7}$. This design consists of a micro-photodiode array three $\mathrm{mm}$ in diameter and $50 \mu \mathrm{m}$ in thickness, and contains of 7000 micro-electrodes in a checker-board pattern configuration. The microphotodiode array is designed to be both insulating and permeable to light ${ }^{4-49}$. The prosthesis also has an external power source to provide enough energy to the subretinal implant. Various animal models with retinal degenerations were used to test and define the micro-photodiode array parameters in order to adequately stimulate ganglion cells. After successfully defining these parameters, the latter were assigned to the development of new prostheses to be implanted for further animal experimentation ${ }^{50-52}$. The goals of the latter experiments were to detect stimulation of the visual cortex (as a result of prosthetic stimulation) and to test the long-term bio-compatibility as well as stability of the prosthesis.

After 14 months of implantation, angiography and histology revealed that there were no rejection reactions or inflammation in the tested targets ${ }^{46}$. Zrenner et al. also recorded the cortical evoked potentials during light flash experiments. Currently there is one relatively successful human trial where the blind patient is capable of indentifying letters and a clock face, and of moving around a room independently ${ }^{53}$. Unlike with epiretinal implants, this approach does not need an external camera. New versions of this prosthesis are concentrating on gathering the device components beneath the skin and providing energy as well as data to the prosthesis through the skin via an external device ${ }^{53}$. The new devices are expected to bring the ability to recognize objects, read letters and recognize people $^{65}$.

A third type of subretinal prosthesis is being developed by Rizzo and Wyatt et al., a research effort that goes by the name Boston Retinal Implant Project ${ }^{54,55}$. As can be seen in figure six, the prosthesis consists of a micro-fabricated thin-film electrode array, a controller microchip, power supply components and power and data receiver coils. The controller microchip and the power supply components are on one module and the power and data receiver coils as well as electrode array are placed on other module. These two modules are connected by a biocompatible flex substrate. The energy and data of the prosthesis is transferred wirelessly via the power and data coils by inductive coupling ${ }^{55}$.

However, this is just the first generation of the prosthesis and it has some significant disadvantages such as ${ }^{55}$ :

- Small receiver coil, which makes the data and power transfer more difficult

- The silicon coating is not suitable for a long term trial (e.g. 10 or 15 years) 
- The implantation procedure is very difficult as the coil is in the way

- There is little data about the long term survivability of the electrode array

Hence, the second generation of the prosthesis was developed in order to overcome the drawbacks of the first generation ${ }^{55}$ :

- A larger coil is used, which is also more comfortable to the eye.

- The circuitry is enclosed in a hermetic, ceramic case.

- The electrode array is inserted in its own quadrant in a manner which allows for easier in implanting.

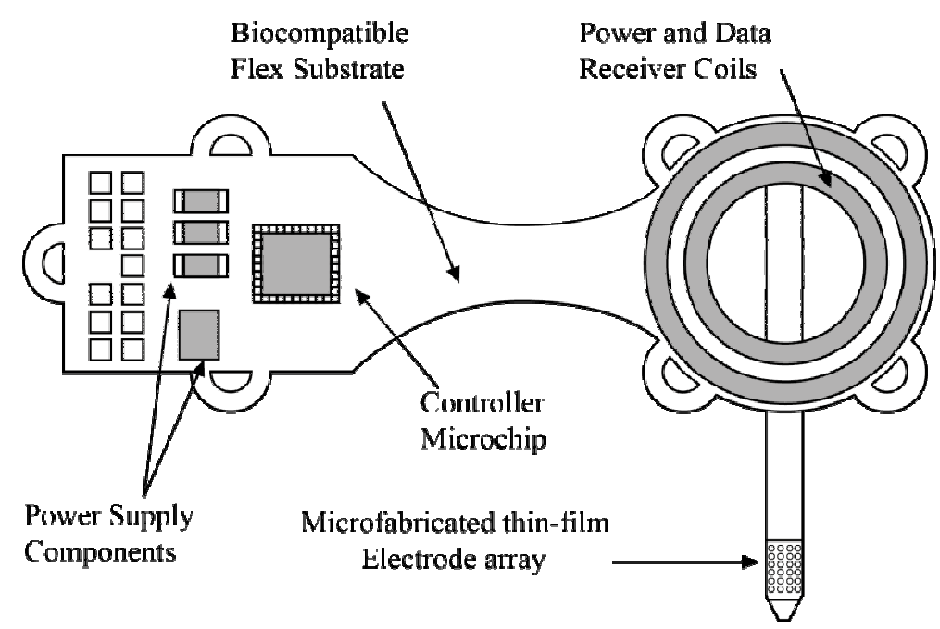

Fig. 6. The first generation of the retinal prosthesis ${ }^{54}$

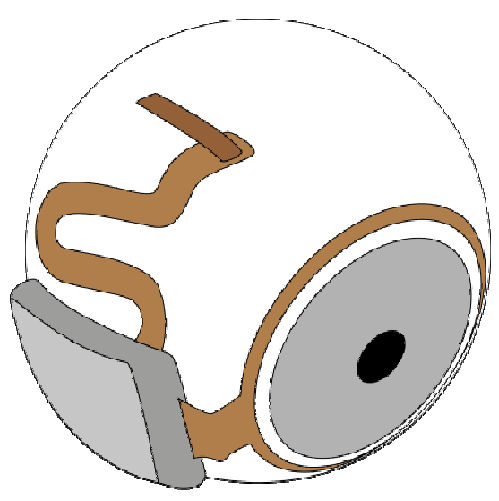

Fig. 7. The hermetic implant in the second generation of the prosthesis ${ }^{55}$

As can be seen in figure seven, the coil is wound on a steel sphere so it can match the curvature of the eye. There are two coils in which the primary coil is used for power telemetry and the second coil is used for data transferring. The prosthesis also contains a 
CMOS chip to receive incoming stimulation data, which it decodes and uses to stimulate the electrodes. The electrical stimulation is provided to the retinal nerve cells via a microfabricated array of sputtered iridium oxide film (SIROF) electrodes. By encasing and placing the electronic components outside the eye, this approach takes advantage of no attachment in the subretinal space therefore there is no need for the eye's natural forces to hold the retina against the choroid ${ }^{54}$.

The prosthesis has been tested on two Yucatan mini-pigs. The result shows consistency in electrode response and good stability over long-term pulsing ${ }^{54}$. Human testing has also been conducted in a series of six experiments. The prosthesis provides very small electrical currents to the retina. Some patients are capable of seeing and distinguishing small spots of light, and perceiving lines in spite of the fact that they have been classified as legally-blind for decades ${ }^{56}$.

On the downside, some negative effects were reported in connection with the conjunctiva. The angle formed by the meeting of the conjunctiva and the coil edge cause tension in the thin conjunctiva. Besides that, the hermetic case is placed too far on the anterior side of the eye, increasing the tension in the conjunctiva. The suggestion for these issues are redesigning the flex circuit that connect the coil and the case as well as the way the case is sutured to the eye to ensure that the case remains in place ${ }^{55}$.

\subsubsection{Advantages and disadvantages of subretinal prostheses}

\section{- Advantages}

The first advantage of the retinal prosthesis is that the remaining retinal tissues can be used for information processing ${ }^{57}$. Apart from advantages pertaining to information compatibility with the rest of the visual system, using intact retinal circuits helps lessen the need for external processing and hence decreases the amount of power needed.

Secondly, it is easier for a subretinal prosthesis to remain in place without leading to retinal detachment. This mechanical stability avoids damaging the surrounding tissue, maintains the prosthesis and retains the consistency of the communication channels between the prosthesis and the intact neurons ${ }^{37}$.

\section{- Disadvantages}

The distribution of the nutritional supply between the choroid and the retina maybe disrupted because of the placement of the device. In other words, if the electrical device is not placed properly, it may accidentally disrupt nutritional flow inside the eye and cause further damage to the retina ${ }^{37}$.

Secondly, power stability is an issue because light absorption in the solar cells is not sufficient nor consistent ${ }^{7}$. Hence, an external power source must be provided to ensure the prosthesis functions reliably.

Thirdly, the inter-neurons on which the prosthesis is relying (i.e. bipolar and amacrine cells) might be heavily re-organized as a consequence of retinal damage, and as a consequence the information conveyed to ganglion cells might be uninterpretable. 


\subsection{Software aspect}

There are several prosthetic approaches that focus on software aspects in order to provide visual restoration to the blind. One approach consists of simulation software that converts images or video sequences into neural spikes. The simulation software can be integrated with hardware solutions to improve the quality as well as the precision of the signals conveyed to intact neurons. In a more conventional way, simulation efforts can be used for scientifically elucidating the structure and function of the retina. The results of these studies can be used in the improvement of hardware designs.

One recent effort in this direction is Virtual Retina, a model that can be used to simulate the retina based on biological constraints ${ }^{58}$. The input of this model is a video sequence and the output is a set of neural spikes, which replicate those produced by retinal ganglion cells. The goal of this software is to simulate a large number of electrical stimulations (up to 100,000 neurons) within a reasonable time ${ }^{58}$.

The model is a layered structure with three main stages:

- The first stage consists of simulating the Outer Plexiform Layer (OPL). At this stage, two cell types are simulated: photoreceptors and horizontal cells. The output from this stage is band-pass excitatory current which is transferred to bipolar cells in next stage.

- The second stage is an implementation of non-linear contrast gain control, in an attempt to simulate the functionality of bipolar cells.

- The last stage simulates the Inner Plexiform layer (IPL) and generates ganglion cell outputs. An excitatory current is fed to ganglion cells after being shaped by the spatio-temporal computations thought to be implemented by the chemical and electrical interactions in the IPL. The model for ganglion cells is a discrete set of integrate-and-fire cells encompassing the visual field. The specific cell types modeled here are either X-type or Y-type.

In order to test the validity of the model's outputs, experiments were conducted on both static and video images. In the first experiment, as can be seen in figure eight, the output reveals increased image details thanks to contrast adjustment, resulting from the model's contrast gain control mechanism ${ }^{58}$. 


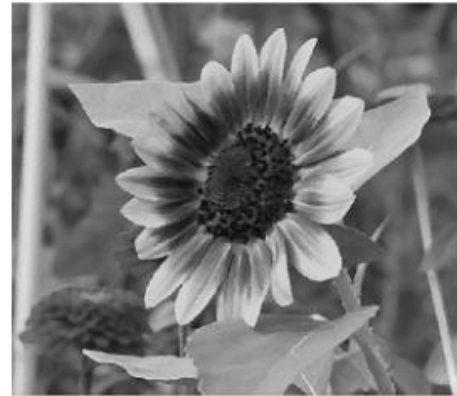

(A)

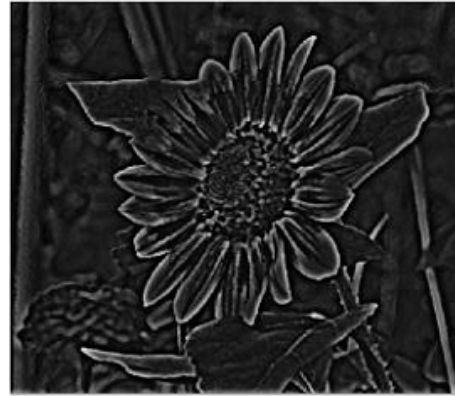

(C)

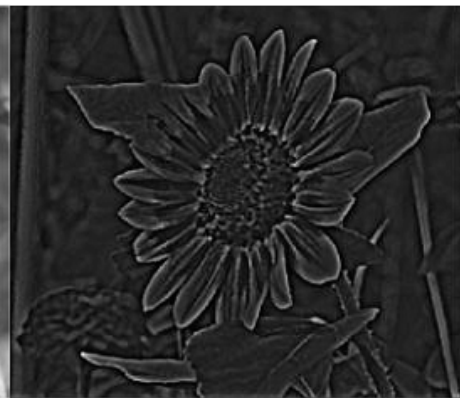

(B)

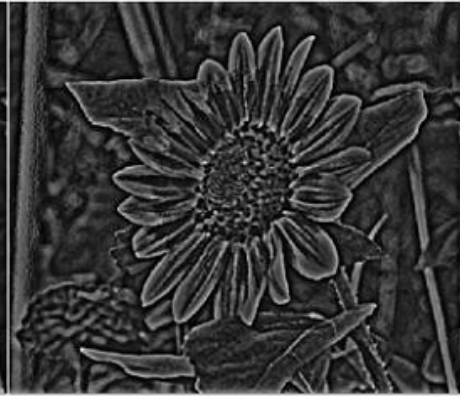

(D)

Fig. 8. The effect of contrast gain control ${ }^{58}$

(A): Input; (B): Linear Output, (C): No feedback; (D): With feedback
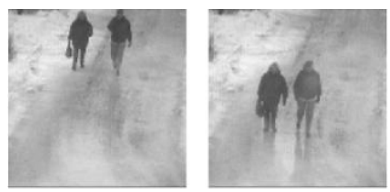

Input Images: $L(x, y, t)$
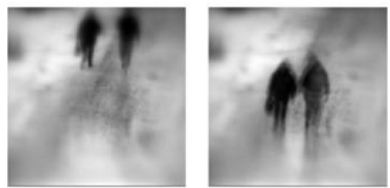

Receptors: $\mathrm{C}(\mathrm{x}, \mathrm{y}, \mathrm{t})$
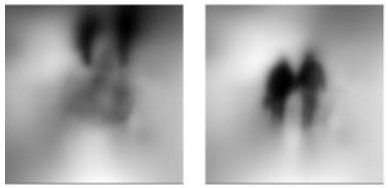

Horizontal cells: $S(x, y, t)$
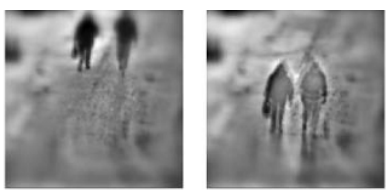

Current on Bip. cells: $\mathrm{I}_{\mathrm{OPL}}(\mathrm{x}, \mathrm{y}, \mathrm{t})$
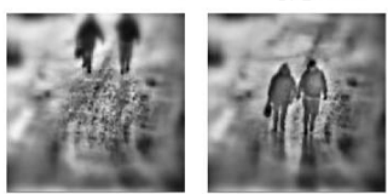

Bipolar cells: $\mathrm{V}_{\mathrm{Bip}}(\mathrm{x}, \mathrm{y}, \mathrm{t})$
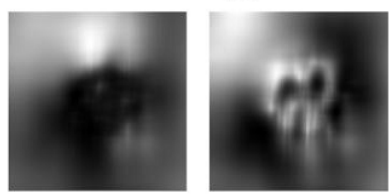

Adaptation in Bip. cells: $g_{A}(x, y, t)$

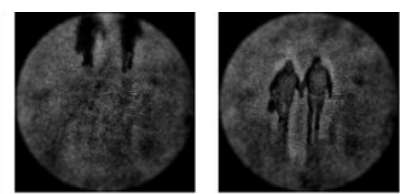

X ON cells (spikes)

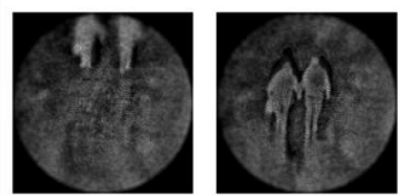

X OFF cells (spikes)
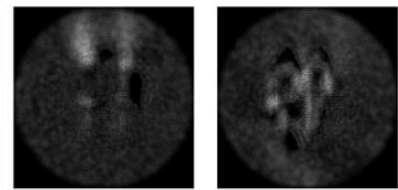

Y OFF cells (spikes)

Fig. 9. Output images from each stage of the virtual retina ${ }^{58}$ 
In the second experiment, as can be seen in figure nine, a video was fed to the model and the output at each stage was evaluated. It is reported that the model demonstrates close correspondence to the outputs from each layer in the biological retina. For example, output images exhibit peripheral blurring due to the foveated structure of the retina. The outputs from the OPL stage also exhibit some saliency for edges and motion ${ }^{58}$. The authors also stated that the model combines two gain control mechanisms, one temporal and the other spatial. The model uses the concept of retinal feedback to improve the contrast of input images as what is claimed to happen in the biological retina. In summary, the model allows for large scale simulations of spiking neurons (up to 100,000) and demonstrates two major properties of the retina: dynamic gain control and spatial non-linearity of cat $\mathrm{Y}$ cells ${ }^{58}$.

The second major simulation model is designed to help researchers get a better understanding of the retina in order to improve the design of the retinal prostheses ${ }^{59}$. The main goal of this model is to discover the mechanisms of information processing inside the retina at the level of individual neurons and the level of entire circuits.

A computer model of the retina is provided as can be seen in the figure ten

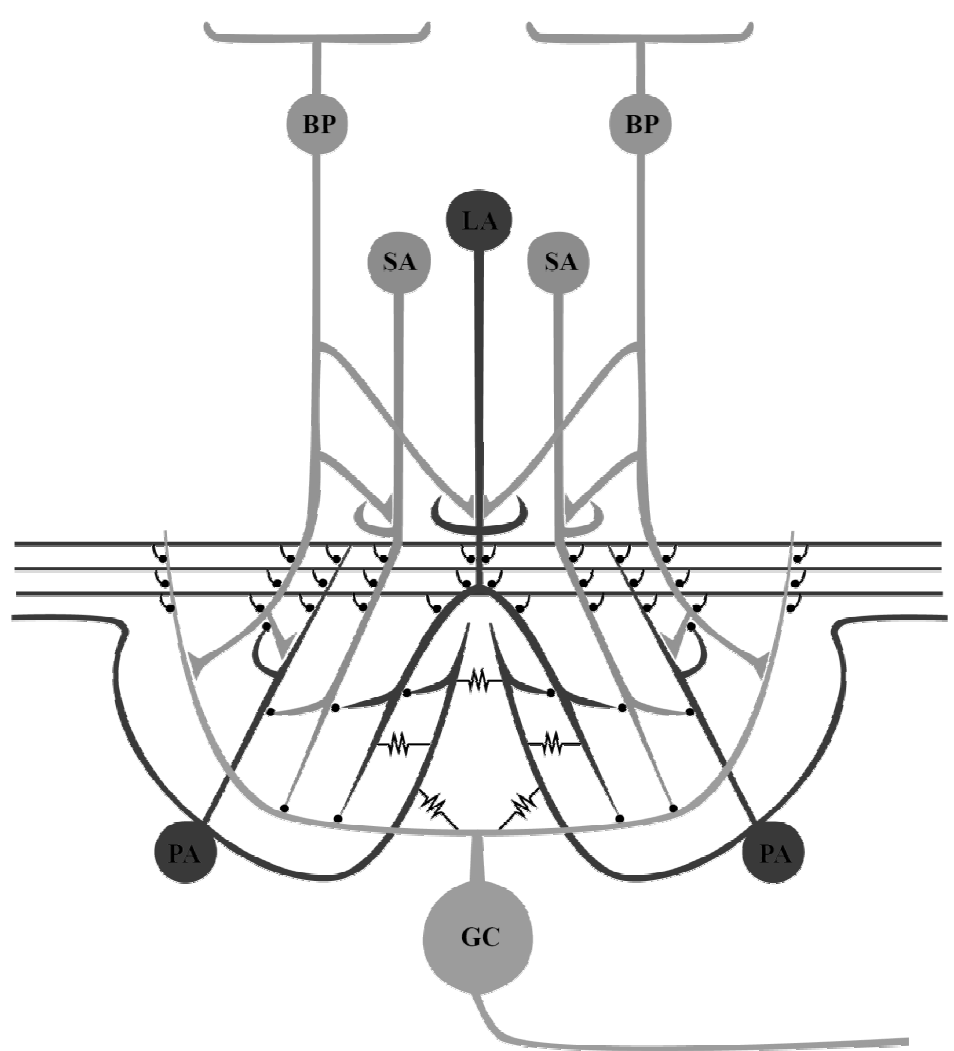

Fig. 10. Computer model of the retina ${ }^{59}$ 
There are five cell types in this model: bipolar cell (BP), small amacrine cell (SA), large amacrine cell (LA), polyaxonal amacrine cell (PA) and alpha ganglion cell (GC). The model has a minimum number of amacrine cell types to simulate the basic functions of the retina when responding to light. The connections in this model are categorized into three types: excitation, long inhibition and long-range axon-mediated feedback ${ }^{59}$. This retinal model exhibits nonlinear properties analogous to the biological retina and provides evidence for the size dependence of retinal oscillations. The oscillations observed in this retinal model are similar to the oscillations observed in cat retina. The model recreates the stimulus selectivity of retinal oscillations ${ }^{59}$.

The two models briefly discussed above are implemented by simulating a subset of retinal cells and the results are encouraging enough to motivate other researchers to build other retinal models with specific purposes. As already mentioned, one major goal of retinal models is to improve the hardware design of retinal prosthesis, hence simulation modeling has an important role to play in neural prosthesis research.

\section{Conclusion}

So far, a significant number of neural prostheses have been developed and tested with the hope to bring vision back to the blind. Although several encouraging results have been obtained the goal of completely restoring vision is still far out of reach because of several different issues. For example, retinal science is a relatively young field and therefore knowledge limitations (with regards to the structure and function of the retina) constitute one major obstacle that needs to be overcome in order to design more effective prostheses. Hardware constraints are believed to be solvable in the near future with the developing rate of current technologies. Somewhat overlooked, software aspects (e.g. image processing, signal processing, compression, coding, information channels, etc.) need renewed focus in order obtain maximum benefit from the existing hardware.

\section{Acknowledgements}

This research was supported by funding from the Fundamental Research Grant Scheme (FRGS) Malaysia, The University of Nottingham Malaysia Campus and The University of Nottingham. 


\section{References}

1. Weiland, J.D., Liu, W. \& Humayun, M.S., 2005. Retinal prosthesis. Annual review of biomedical engineering, 7, pp. 361-401.

2. Weisz JM, O'Connell SR, Bressler NM. 2000. Treatment guidlines for age-related macular degeneration based upon results from the macular photocoagulation study. In Macular Surgery, ed. H Quiroz-Mercado, DV Alfaro III, PE Ligget, Y Tano, E de Juan Jr, pp. 201-11. Philadelphia: Lippincott, Williams \& Wilkins.

3. Berson EL. 1993. Retinitis pigmentosa. The Friedenwald Lecture. Invest. Ophthalmol. Vis. Sci. 34(5):1659-76.

4. Sharma RK, Ehinger B. 1999. Management of hereditary retinal degenerations: present status and future directions. Surv. Ophthalmol. 43(5):427-44.

5. Heckenlively JR, Boughman J, Friedman L. 1988. Diagnosis and classification of retinitis pigmentosa. In Retinitis Pigmentosa, ed. JR Heckenlively, p. 21. Philadelphia, PA: Lippincott

6. Matthew Schmolesky. The Primary Visual Cortex. Online resource. Access on 12/12/2010, at http://webvision.med.utah.edu/VisualCortex.html.

7. Javaheri, M. et al., 2006. Retinal prostheses for the blind. Annals of the Academy of Medicine, Singapore, 35(3), pp. 137-44.

8. Dobelle WH. Artificial vision for the blind by connecting a television camera to the visual cortex. ASAIO J 2000; 46:3-9.

9. Uhlig CE, Tanen S, Benner FP, Gerding H. Electrical stimulation of the visual system. From empirical approach to visual prostheses. Ophthalmologe 2001; 98:1089-96.

10. Weiland JD, Humayun MS. Past, present, and future of artificial vision. Artif Organs 2003; 27:961-2.

11. Foerster O. Beitrage zur pathophysiologie der sehbahn und der spehsphare. J Psychol Neurol 1929; 39:435-63. In Retinal prostheses for the blind. Javaheri, M. et al., 2006. Annals of the Academy of Medicine, Singapore, 35(3), pp. 137-44.

12. Brindley G. The number of information channels needed for efficient reading. J Physiol 1965; 177:44.

13. Margalit E, Maia M, Weiland JD, Greenberg RJ, Fujii GY, Torres G, et al. Retinal prosthesis for the blind. Surv Ophthalmol 2002; 47:335-56.

14. Maynard EM, Nordhausen CT, Normann RA. The Utah intracortical electrode array: a recording structure for potential brain-computer interfaces. Electroencephalogr Clin Neurophysiol 1997; 102:228-39.

15. Uematsu S, Chapanis N, Gucer G, Konigsmark B, Walker AE. Electrical stimulation of the cerebral visual system in man. Confin Neurol 1974; 36:113-24.

16. Troyk P, Bak M, Berg J, Bradley D, Cogan S, Erickson R, et al. A model for intracortical visual prosthesis research. Artif Organs 2003; 27:1005-15.

17. Veraart, C. et al., 1998. Visual sensations produced by optic nerve stimulation using an implanted self-sizing spiral cuff electrode. Brain research, 813(1), pp. 181-6.

18. Veraart C, Wanet-Defalque MC, Gerard B, Vanlierde A, Delbeke J. Pattern recognition with the optic nerve visual prosthesis. Artif Organs 2003;27:996-1004.

19. Oozeer, M. et al., 2006. A model of the mammalian optic nerve fibre based on experimental data. Vision research, 46(16), pp. 2513-24.

20. Wikipedia, Visual Prosthesis. Online Resource. Access on 12/12/2010. At http://en.wikipedia.org/wiki/Visual_prosthesis.

21. Weiland JD, Liu W, Humayun MS. Retinal prosthesis. Annu Rev Biomed Eng 2004.

22. Humayun MS, de Juan E Jr, Dagnelie G, Greenberg RJ, Propst RH, Phillips DH. Visual perception elicited by electrical stimulation of retina in blind humans. Arch Ophthalmol 1996; 114:40-6. 
23. Humayun MS, de Juan E Jr, Weiland JD, Dagnelie G, Katona S, Greenberg R. Pattern electrical stimulation of the human retina. Vision Res 1999; 39:2569-76.

24. Humayun MS, Weiland JD, Fujii GY, Greenberg R, Williamson R, Little J, et al. Visual perception in a blind subject with a chronic microelectronic retinal prosthesis. Vision Res 2003; 43:2573-81.

25. Lakhanpal R, Yanai D, Weiland JD, Fujii GY, Caffey S, Greenberg RJ, et al. Advances in the development of visual prostheses. Curr Opin Ophthalmol 2003; 14:122-7.

26. David Rose. Surgeons give hope to blind with successful 'bionic eye' operations. Online Resource. Access on 12/12/2010, at http://www.timesonline.co.uk/tol/news/uk/health/article3790683.ece

27. Rizzo JF 3rd, Wyatt J, Loewenstein J, Kelly S, Shire D. Methods and perceptual thresholds for short-term electrical stimulation of human retina with microelectrode arrays. Invest Ophthalmol Vis Sci 2003; 44:5355-61.

28. Rizzo JF 3rd, Wyatt J, Loewenstein J, Kelly S, Shire D. Perceptual efficacy of electrical stimulation of human retina with a microelectrode array during short-term surgical trials. Invest Ophthalmol Vis Sci 2003; 44:5362-9.

29. Eckmiller RE. Learning retina implants with epiretinal contacts. Ophthalmic Res 1997; 29:281-9.

30. Walter P, Szurman P, Vobig M, Berk H, Ludtke-Handjery HC, Richter H, et al. Successful long-term implantation of electrically inactive epiretinal microelectrode arrays in rabbits. Retina 1999; 19:546-52.

31. Eckmiller RE, Hornig R, Gerding H, Dapper M, Böhm H. Test technology for retina implants in primates [ARVO abstract]. Invest Ophthalmol Vis Sci 2001; 42:S942.

32. Laube T, Schanze T, Bolle I, Brockmann C, Bornfeld N. Long-term follow-up of epidural electrodes in minipigs for registration of visually evoked cortical potentials (VEP) and electrically evoked cortical potentials (EEP) [ARVO abstract]. Invest Ophthalmol Vis Sci 2002. Abstract nr 4457.

33. Gerding HR, Hornig R, Eckmiller R, Dapper M, Taneri S, Uhlig CE, et al. Implantation, mechanical fixation, and functional testing of epiretinal multimicrocontact arrays (MMA) in primates [abstract]. Invest Ophthalmol Vis Sci 2001; 42 (Suppl):S814.

34. Eckmiller RE, Neumann D, Baruth O. Tunable retina encoders for retina implants: why and how. J Neural Eng 2005; 2:S91-S104.

35. Eckmiller RE, Baruth O, Neumann D. Learning retina encoder RE: results from dialog-based tuning in humans with normal vision [ARVO abstract]. Invest Ophthalmol Vis Sci 2005. Abstract nr 5266.

36. Piyathaisere DV, Margalit E, Chen SJ, et al. Effects of short-term exposure to heat on the retina [abstract]. Invest Ophthalmol Vis Sci 42(Suppl):S814, 200.

37. Weiland, J.D. \& Humayun, M.S., 2006. Intraocular retinal prosthesis. IEEE Engineering in Medicine and Biology Magazine, 25(5), pp. 60-66.

38. Chow AY, Chow VY, Packo KH, Pollack JS, Peyman GA, Schuchard R. The artificial silicon retina microchip for the treatment of vision loss from retinitis pigmentosa. Arch Ophthalmol 2004; 122:460-9.

39. Chow AY, Peachey NS. The subretinal microphotodiode array retinal prosthesis [comment]. Ophthalmic Res 1998; 30:195-8.

40. Peyman G, Chow AY, Liang C, Chow VY, Perlman JI, Peachey NS. Subretinal semiconductor microphotodiode array. Ophthalmic Surg Lasers 1998; 29:234-41.

41. Chow AY, Pardue MT, Perlman JI, Ball SL, Chow VY, Hetling JR, et al. Subretinal implantation of semiconductor-based photodiodes: durability of novel implant designs. $\mathrm{J}$ Rehabil Res Dev 2002; 39:313-21. 
42. Chow, a Y. \& Chow, V.Y., 1997. Subretinal electrical stimulation of the rabbit retina. Neuroscience letters, 225(1), pp. 13-6.

43. Zrenner, E., 2002. Will retinal implants restore vision? Science (New York, N.Y.), 295(5557), pp. 1022-5.

44. Eckhorn, R. et al., 2006. Visual resolution with retinal implants estimated from recordings in cat visual cortex. Vision research, 46(17), pp. 2675-90.

45. Dobelle WH. Artificial vision for the blind by connecting a television camera to the visual cortex. ASAIO J 46:3-9, 2000.

46. Dobelle WH, Mladejovsky MG. Phosphenes produced by electrical stimulation of human occipital cortex, and their application to the development of a prosthesis for the blind. $\mathrm{J}$ Physiol 243:553-76, 1974.

47. Dobelle WH, Mladejovsky MG, Evans JR, et al. "Braille" reading by a blind volunteer by visual cortex stimulation. Nature 259:111-2, 1976.

48. Dowling JE. The retina: an approachable part of the brain. London, Belknap Press, 1987, pp. 81-123.

49. Dougherty SH, Simmons RL. Infections in bionic man: the pathobiology of infections in prosthetic devices-Part II. Curr Probl Surg 19:265-319, 1982.

50. Eckmiller R. Learning retina implants with epiretinal contacts. Ophthalmic Res 29:281-9, 1997

51. Evans JR, Gordon J, Abramov I, et al. Brightness of phosphenes elicited by electrical stimulation of human visual cortex. Sens Processes 3:82-94, 1979.

52. Foerster O: Beitrage zur pathophysiologie der sehbahn und der spehsphare. J Psychol Neurol 39:435-63, 1929. In Retinal prostheses for the blind. Javaheri, M. et al., 2006. Annals of the Academy of Medicine, Singapore, 35(3), pp. 137-44.

53. Neil Bowdler. Implanted chip 'allows blind people to detect objects'. Online Resource. Access on 12/12/2010, at http://www.bbc.co.uk/news/health-11670044

54. Sweitzer, R. \& Cogan, S., 2004. The Retinal Implant Project. Current, pp. 1-13.

55. Kelly, S.K. et al., 2009. The Boston retinal prosthesis: A 15-channel hermetic wireless neural stimulator. 2009 2nd International Symposium on Applied Sciences in Biomedical and Communication Technologies, pp. 1-6.

56. The Boston Retinal Implant. Human Testing. Online Resource. Access on 12/12/2010, at http://www.bostonretinalimplant.org/humantest.php?fontsize=normal\&hicontrast=

57. Sachs, H.G. \& Gabel, V.-P., 2004. Retinal replacement-the development of microelectronic retinal prostheses-experience with subretinal implants and new aspects. Graefe's Archive for Clinical and Experimental Ophthalmology, 242(8), pp. 717-723.

58. Wohrer, A. \& Kornprobst, P., 2009. Virtual Retina: a biological retina model and simulator, with contrast gain control. Journal of computational neuroscience, 26(2), pp. 219-49.

59. Kenyon, G.T. et al., 2005. Models of the Retina with Application to the Design of a Visual Prosthesis. Los Alamos Science, (29), pp. 110-123.

60. Biological machines. Wikibooks. License under GFPL. Online Resource. Access on 12/12/2010 at http://en.wikibooks.org/wiki/File:Retina.jpg

61. Jutta Scheibe, Morten Lyhne. Optic Nerve Prosthesis Concept. Catholic University of Louvain, Belgium.

62. Aaron Saenz. Argus III - The Artificial Retina is Near! Online Resource. Access on 12/12/2010, at http://singularityhub.com/2010/02/25/argus-iii-the-artificial-retina-is-near/. Image credit: Lawrence Livermore National Labs. 
63. Kim, S.Y. et al., 2002. Morphometric Analysis of the Macula in Eyes With Disciform AgeRelated Macular Degeneration. RETINA, 22(4).

64. Veraart C, Wanet-Defalque MC, Delbeke J et al. Assessment of the MIVIC optic nerve visual prosthesis [abstract]. Invest Ophthalmol Vis Sci 2001; 42 (Suppl.):942.

65. Institute for Ophthalmic Research, Retina Implant Project. Online Resource. Access on 12/12/2010, at http://www.eye.uni-tuebingen.de/retina-implant-project. 